\title{
Reproductive Outcomes in Patients with Severe Oligospermia Undergoing Intracytoplasmic Sperm Injection using Testicular Versus Ejaculated Spermatozoa
}

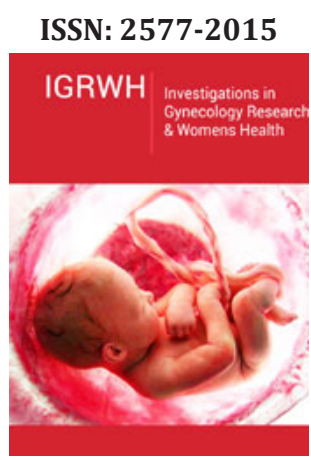

*Corresponding author: Mahmoud HM, Department of Obstetrics/Gynecology, Faculty of Medicine, University of Alexandria, Madina Fertility Center, Alexandria, Egypt

Submission: 海 June 15, 2019

Published: 笽June 26, 2019

Volume 3 - Issue 1

How to cite this article: Mahmoud H, Hebisha S. Reproductive Outcomes in Patients with Severe Oligospermia Undergoing Intracytoplasmic Sperm Injection using TesticularVersus Ejaculated Spermatozoa. Invest Gynecol Res Women's Health.3(1). IGRWH.000551.2019. DOI: 10.31031/IGRWH.2019.03.000551

Copyright@ Mahmoud MH, This article is distributed under the terms of the Creative Commons Attribution 4.0 International License, which permits unrestricted use and redistribution provided that the original author and source are credited.

\author{
Mahmoud HM ${ }^{1,2 *}$ and Hebisha SA ${ }^{1,2}$ \\ ${ }^{1}$ Department of Obstetrics/Gynecology, Faculty of Medicine, University of Alexandria, Egypt \\ ${ }^{2}$ Madina Fertility Center, Alexandria, Egypt
}

\begin{abstract}
Background: In infertile men with severe oligospermia (sperm concentration $<5$ million $/ \mathrm{mL}$ ) undergoing ICSI the choice between using ejaculated sperm or testicular sperm remains controversial. Testicular sperm have shown lower levels of DNA damage compared with ejaculated spermatozoa from the same individuals.
\end{abstract}

Objective: To compare intracytoplasmic sperm injection (ICSI) outcome in patients with severe oligospermia using testicular versus ejaculated spermatozoa.

Design: Retrospective cohort study.

Setting: Medina Fertility Center

Materials and Methods: Patients with severe oligospermia who underwent ICSI cycles with either testicular or ejaculated sperm between January 2015 and December 2017 were included in this study. 205 couples met the study criteria after exclusion of cycles with mixed sperm sources. Patients were divided into 2 subgroups according to the source of sperm used in intracytoplasmic sperm injection (ICSI) cycle; (Group A) included 104 patients in which ejaculated sperm were injected while (Group B) included 101 patients where fresh testicular sperm were extracted (TESE) and injected. The medical records of the included couples were reviewed and tabulated regarding demographic data, reproductive history, hormonal profiles and different semen parameters. The reproductive outcomes; fertilization rate, number of high-quality embryos, and pregnancy rate were evaluated and compared in both groups.

Results: There was no significant difference in the fertilization rate between the two subgroups (group A $67.93 \%$, group B $68.01 \%, p=0.960$ ), the total number of high-quality embryos tended to be higher in the testicular sperm group, but the difference did not reach statistical significance (187 vs $154, \mathrm{P}=0.084$ ). The clinical pregnancy rate was significantly higher in group B where testicular spermatozoa were injected (49.50\% vs $35.57 \%$, $\left.\mathrm{p}=0.044^{*}\right)$.

Conclusion: In patients with severe oligospermia, when both sources of spermatozoa are available (i.e. ejaculate or testicular) for the ICSI procedure, TESE should be considered as it offers higher pregnancy rates

Keywords: Infertility; Severe oligospermia; Intracytoplasmic sperm injection; Testicular sperm extraction (TESE)

\section{Introduction}

Male factor is an important cause of infertility, isolated male factor accounts for $25 \%$ of cases of infertility. The development of the ICSI procedure and surgical sperm retrieval techniques have been of great benefit to achieve pregnancy in men with such conditions [1]. Cases with sufficient number of sperms for injection in the ejaculate (including cases with severe oligospermia, defined most commonly in literature as sperm count below 5 million per $\mathrm{ml}$ ) [2,3] are injected using ejaculate sperm. Since the testicular sperm may not reach the same maturity as the ejaculated sperm which completes its maturation during its transit in the genital tract, thus with normal semen parameters the fertilization potential and the success rates are higher with ejaculate sperm than with testicular sperm [4]. Evidence shows that infertile men have more sperm DNA damage than fertile men [5]. Apparently, this is more profound in oligospermic men as Moskovtsev et al. [6] analyzed semen from more than 
2500 men and found that low sperm counts are associated with higher DNA damage [6]. The American Society of Reproductive Medicine (ASRM) Practice Committee has reported that measuring the DNA fragmentation might be helpful in predicting the outcome of ICSI and Esteves et al suggested that integrity testing should be integrated in the basic semen analysis $[7,8]$ Studies have shown that in men with high DNA fragmentation in their ejaculate sperm, testicular sperm tended to have better quality with lower DNA damage [9]. Researchers have shown that with increased DNA damage, there is reduction in the fertility potential, as well as a drop in most of the outcome parameters of ART, namely the embryo quality, the implantation rate, the clinical pregnancy rate, the ongoing pregnancy rate, and the live birth rate $[9,10]$.

The exact cause for sperm DNA fragmentation remains unclear, but several factors have been accused such as: gonadotoxic exposure to pollutants, varicocele, infections, systemic diseases and advanced age [11-13]. Studies have shown that sperm can be exposed to oxidative stress leading to damage to its DNA while it is being transported in the male genital tract $[14,15]$. This is supported by the fact that the process of chromatin condensation is still in progress during epididymal transport and that epididymal epithelial cells can produce excessive amounts of free oxygen radicals when exposed to unfavorable conditions [15,16]. Multiple strategies have been proposed to improve the sperm DNA integrity like the use of oral antioxidants for few months before ICSI, varicocelectomy surgery, the use of multiple ejaculates before ICSI, or the use of "intracytoplasmic morphologically selected sperm injection"(IMSI) [17-19]. Unfortunately, none of these strategies has been proven to alleviate the negative effect of DNA damage on ICSI success rates [7]. In infertile men with oligospermia and high ejaculate DNA damage, testicular sperm has been proven to have markedly less DNA fragmentation [20]. This has led to the postulation that in oligospermic men, the use of the testicular sperm with its higher DNA integrity might yield higher success rates.

\section{Materials and Methods}

Design: Retrospective cohort study

Setting: Patients with severe oligospermia who underwent ICSI/ fresh ET cycles in Medina Fertility Center using either ejaculate or testicular sperm between January 2015 and December 2017 were included in this study. 205 couples met the selection criteria.

\section{Inclusion criteria}
i. Sperm count $<5$ millionml
ii. Normal HSG and normal transvaginal ultrasound
iii. Women age $>40$ years
iv. Long agonist or antagonist protocols of stimulation

\section{Exclusion criteria}
i. Cycles with mixed sperm sources
ii. Obese females with $\mathrm{BMI}<30 \mathrm{~kg} / \mathrm{m}^{2}$
iii. Poor responders

\section{iv. Freeze all cycles}

v. Absence of good quality embryos for transfer

The medical records of the included couples were reviewed and tabulated regarding demographic data, reproductive history, hormonal profiles, different semen parameters, cycle performance and outcome.

\section{Analysis of semen parameters}

i. Basic sperm parameters (count, motility and morphology) were evaluated in all cases according to the World Health Organization criteria standards (WHO, 2010).

ii. Patients were divided into 2 groups according to the source of sperm used:

A. (Group A): included 104 patients in whom ejaculate sperm was used for injection

B. (Group B): included 101 patients who underwent TESE and fresh testicular sperm were injected.

\section{Outcome analysis}

Cases in both groups were compared as regards: Demographic data, hormonal profile on initial work up, different parameters of the semen analysis, number of oocytes retrieved, number of mature Metaphase II oocytes, fertilization rate, number of high quality embryos, chemical and clinical pregnancy rates where chemical pregnancy was defined as a positive pregnancy test with failure to identify a gestational sac two weeks after the pregnancy test, while clinical pregnancy rate was defined as demonstration of a gestational sac with a positive heart beat two weeks after the pregnancy test (30 days after oocyte retrieval). Statistical analysis of the obtained data was carried out using standard SPSS software for Windows version 20. To assess the significance of the differences in the quantitative characteristics, the Student's t-test and F-Fisher criteria were applied, the differences were considered significant at p-value $<.05$ Qualitative data were described using number and percent and was compared using Chi square test. Abnormally distributed data was expressed using Median (Min.-Max.) and was compared using Mann Whitney test. Normally distributed quantitative data was expressed as Mean \pm SD and compared using student t-test. While abnormally distributed data was expressed using Median (Min.-Max.) and was compared using Mann Whitney test.

\section{Result}

Both groups showed no significant difference as regards the demographic data and the pre-ICSI hormonal profile as well as the semen parameters (Tables 1 and 2). The number of oocytes retrieved per case and the number of mature oocytes were not significantly different between both groups. The fertilization rate was nearly identical in both groups $(67.93 \%$ vs $68.01, \mathrm{p}=0.96)$ (Table 3). The number of high-quality embryos was higher in the testicular sperm group than in the ejaculate sperm group (187 vs 154), but this difference did not reach statistical significance $(p=0.084)$. The total number of embryos transferred was very 
similar between both groups (214 vs 215). Furthermore, there was no significant difference between both groups as regards the numbers of embryos transferred per case $(\mathrm{p}=0.561)$ (Table 4). The clinical pregnancy rate was $49.5 \%$ in the testicular sperm group which was significantly higher than the $35.57 \%(p=0.044)$ observed in the ejaculate sperm group. Both groups had 5 cases of chemical pregnancies. This similarity did not affect the overall pregnancy rate which was significantly higher in the testicular sperm group than the ejaculate sperm group ( $54.45 \%$ vs $40.38 \%$, $\mathrm{p}=0.0442$ ) (Table 5).

Table 1: Comparison between the two studied groups regarding the demographic data and hormonal profile.

\begin{tabular}{|c|c|c|c|}
\hline & Group A Ejaculate Sperm (n=104) & \multicolumn{2}{|c|}{ Group B Testicular Sperm (n=101) } \\
\hline Age(years) & \multicolumn{3}{|c|}{0.132} \\
\hline Male & $36.3 \pm 6.9$ & $37.7 \pm 7.2$ & 0.284 \\
\hline Female & $29.4 \pm 5.4$ & $40.2 \pm 5.4$ & 0.562 \\
\hline Years of infertility Hormonal Profile & $4(0-17)$ & $2.1(0-9.5)$ & 0.121 \\
\hline AMH & $3(0.1-8.5)$ & $6.3(2.3-26.2)$ & 0.325 \\
\hline FSH & $6(1-26.5)$ & $7.9 \pm 2.9$ & 0.783 \\
\hline LH & $7.8 \pm 3$ & $370(116-924)$ & 0.497 \\
\hline Testosterone & $380(2.3-990)$ & $5.7(1.3-15.4)$ & 0.298 \\
\hline Free Testosterone & $6.1(0.5-17.3)$ & $8.3(1.8-39)$ & 0.364 \\
\hline
\end{tabular}

*: Statistically significant at $\mathrm{p}<0.05$

Table 2: Comparison between the two studied groups according to different semen parameters.

\begin{tabular}{|c|c|c|c|}
\hline & Group A Ejaculate Sperm (n=104) & \multicolumn{2}{|c|}{ Group B Testicular Sperm (n=101) } \\
\hline Semen Abnormality & \multicolumn{3}{|c|}{ P } \\
\hline Normal & $21(20.2 \%)$ & $23(22.8 \%)$ & 0.638 \\
\hline Teratospermia & $83(79.8 \%)$ & $3(0.2-6.6)$ & 0.826 \\
\hline Volume $(\mathrm{ml})$ & $3(1-8)$ & $4.1(0.3-4.9)$ & 0.073 \\
\hline Conc. $(\times 106 / \mathrm{ml})$ & $3.8(0.1-4.6)$ & $3.5(0-46)$ & 0.75 \\
\hline Motile $\%$ & $5.2(1-45)$ & 0.653 \\
\hline
\end{tabular}

*: Statistically significant at $\mathrm{p}<0.05$

Table 3: Comparison between the two studied groups according to the number of oocytes retrieved, their maturity, and the fertilization rate.

\begin{tabular}{|c|c|c|c|}
\hline & Group A Ejaculate Sperm (n=104) & Group B Testicular Sperm (n=101) & P \\
\hline Oocyte number & $16(3-25)$ & $14(4-28)$ & 0.492 \\
\hline M2 oocytes & $14(2-25)$ & $13(2-26)$ & 0.789 \\
\hline Fertilization rate & $67.93 \%$ & $68.01 \%$ & 0.96 \\
\hline
\end{tabular}

*: Statistically significant at $\mathrm{p}<0.05$

Table 4: Comparison between the two studied groups according to the number of high-quality embryos, number of embryos transferred (total and per case).

\begin{tabular}{|c|c|c|c|}
\hline & $\begin{array}{c}\text { Group A Ejaculate Sperm } \\
(\mathbf{n = 1 0 4 )}\end{array}$ & $\begin{array}{c}\text { Group B Testicular Sperm } \\
\text { (n=101) }\end{array}$ & P \\
\hline Number of high-quality Embryos & 154 & 187 & 0.084 \\
\hline Total number of embryos transferred & 214 & 215 & 0.982 \\
\hline Embryos transferred per case & $11(10.6 \%)$ & $8(7.9 \%)$ & \\
1 embryo & $78(75.0 \%)$ & $19(72.3 \%)$ & 0.561 \\
2 embryos & $13(12.5 \%)$ & $1(1.0 \%)$ & \\
3 embryos & $2(1.9 \%)$ & $1.0 \%)$ & \\
\hline
\end{tabular}

*: Statistically significant at $\mathrm{p}<0.05$ 
Table 5: Comparison between the two studied groups according to pregnancy outcome.

\begin{tabular}{|c|c|c|}
\hline Outcome & $\begin{array}{c}\text { Group A } \\
\text { Ejaculate Sperm } \\
(\mathbf{n = 1 0 4 )}\end{array}$ & $\begin{array}{c}\text { Group B } \\
\text { Testicular Sperm } \\
(\mathbf{n = 1 0 1 )}\end{array}$ \\
\hline Chemical Pregnancy & $5(4.80 \%)$ & $5(4.95 \%)$ \\
\hline Clinical Pregnancy & $37(35.57 \%)$ & $50(49.50 \%)$ \\
\hline Overall Pregnancy & $42(40.38 \%)$ & $0.964 *$ \\
\hline \multirow{2}{*}{$0.0442^{*}$} & $55(54.45 \%)$ \\
\hline
\end{tabular}

*: Statistically significant at $\mathrm{p}<0.05$

\section{Discussion}

Male factor remains a major cause of infertility. ICSI is considered as a final solution for such cases. In male factor cases of infertility, the success rate largely depends on the quality of the sperm selected for injection. Besides its morphology and motility, sperm DNA integrity is a major determinant of the quality of the injected sperm. Cases with high sperm DNA fragmentation index (DFI) have been shown to have poor ICSI outcomes with lower fertilization rate, pregnancy rate, live birth rate, and higher abortion rate [21,22]. Recent studies have shown that the ejaculated and the epididymal spermatozoa have higher DNA damage than the testicular spermatozoa; this can be explained by the fact that testicular spermatozoa are protected by the Sertoli cells from exposure to damaging factors, and the ability of the genital tract to generate excessive reactive oxygen species (ROS) under the effect of infection, high temperature or exposure to environmental pollutants $[12,18,23,24]$. The present study was performed to compare the outcome of ICSI in patients with severe oligospermia using testicular versus ejaculated spermatozoa.

This study found no significant difference in the fertilization rate between the two study groups, this was in agreement with the findings reported by Mehta et al. [20], Abhyankar et al. [25], Arafa et al. [26] and Herrero et al. [27] who all showed that even with the use of sperm with high DFI, fertilization can still be achieved, this is because fertilization and early embryo development are mainly controlled by maternal DNA. Paternal factors, on the other hand are involved in later stages of embryo development which can be manifested in the quality of embryos, the pregnancy rate, and the abortion rate. The current study found the high-quality embryos to be higher in the testicular sperm group, the difference though did not reach statistical significance. This finding was in concordance with the results presented by Arafa et al. [26] and Esteves et al. [28]. As regards the clinical pregnancy rate, it was significantly higher in the testicular sperm group than in the ejaculate sperm group ( $p=0.044$ ). Similar results were reported by Arafa et al. [26], Herrero et al. [27], Esteves et al. [28], Zhang et al. [29] and Mehta et al. [20] performed ICSI with testicular spermatozoa on 24 couples with severe oligospermia who had multiple previous failed cycles with ejaculated sperm; they achieved $50 \%$ clinical pregnancy rate in the first cycle using testicular sperm. A limitation of our study was the use of chemical pregnancy rate-instead of abortion ratewhere both groups had similar rates as this did not identify the cases which had later miscarriages. In their studies, Arafa et al. [26], Herrero et al. [27], Esteves et al. [28] and Zhang et al. [29] reported a significantly higher abortion rate in the ejaculate sperm group and a significantly higher live birth rate in the testicular sperm group. It is important to note that TESE has its own drawbacks. It is an invasive procedure with added cost and added risk of surgical complications-although of very low incidence-including infection, bleeding, testicular atrophy and decreased testosterone production [30]. Furthermore, Turek et al. [31] reported that testicular sperm are more immature and show a slightly higher incidence of aneuploidy in comparison to ejaculated sperm (12\% vs $6 \%$ ). This finding however remains to be confirmed by larger trials, and because of the fact that ICSI is usually performed to patients who are unlikely to achieve natural conception; a minor elevation in the chance of the children having some health issues is an accepted side effect to having an established higher live birth rate [30].

\section{Conclusion}

ICSI using testicular sperm is a promising option for men with severe oligospermia especially those with high sperm DFI. The added cost and minor complications seem to be outweighed by the benefit of the improved reproductive outcome. Furthermore, it could be argued that the more invasive male treatment is opposed by the less invasive female treatment as lower numbers of cycles are needed to achieve live births.

Larger randomized controlled trials are required to validate these findings and to establish whether this treatment strategy can be applied to oligospermic men with high DNA fragmentation alone or to all cases with high DFI regardless of their sperm count. Whether testicular sperm should be used in such cases from the first ICSI trial or be restricted to cases with previous failures should be evaluated in additional studies.

\section{References}

1. Andersen AN, Carlsen E, Loft A (2008) Trends in the use of intracytoplasmic sperm injection marked variability between countries. Hum Reprod Update 14(6): 593-604.

2. Ishikawa T, Kondo Y, Yamaguchi K, Sakamoto Y, Fujisawa M (2008) Effect of varicocelectomy on patients with unobstructive azoospermia and severe oligospermia. BJU Int 101(2): 216-218.

3. Ng PP, Tang MH, Lau ET, Ng LK, Ng EH, et al. (2009) Chromosomal anomalies and Y-microdeletions among Chinese subfertile men in GHONG Kong. Hong Kong Med J 15(1): 31-38.

4. Ku F, Wu C, Hsiao Y, Kang Y (2018) Association of sperm source with miscarriage and take-home baby after ICSI in cryptozoospermia: a meta-analysis of testicular and ejaculated sperm. Andrology 6(6): 882-889.

5. Zini A, Boman J, Belzile E, Ciampi A (2008) Sperm DNA damage is associated with an increased risk of pregnancy loss after IVF and ICSI: Systematic review and meta-analysis. Hum Reprod 23(12): 2663-2668. 
6. Moskovtsev SI, Willis J, White J, Mullen JBM (2009) Sperm DNA damage: correlation to severity of semen abnormalities. Urology 74(4): 789-793.

7. (2015) Practice committee of the American society for reproductive medicine. Diagnostic evaluation of the infertile male: a committee opinion. Fertil Steril 103: 18-25.

8. Esteves SC, Majzoub A, Agarwal A (2017) Despite limitations, sperm DNA fragmentation testing provides unique information complementary to but distinct from semen analysis results. Transl Androl Urol 6(Suppl 4): 377-378.

9. Moskovtsev SI, Jarvi K, Mullen JB, Cadesky KI, Hannam T, et al. (2010) Testicular spermatozoa have statistically significant lower DNA damage compared with ejaculated spermatozoa in patients with unsuccessful oral antioxidant treatment. Fertil Steril 93 (4): 1142-1146.

10. Simon L, Proutski I, Stevenson M, Jennings D, McManus J, et al. (2013) Sperm DNA damage has a negative association with live-birth rates after IVF. Reprod Biomed Online 26(1): 68-78.

11. Schulte RT, Ohl DA, Sigman M, Smith GD (2010) Sperm DNA damage in male infertility: etiologies, assays, and outcomes. J Assist Reprod Genet 27(1): 3-12.

12. Micillo A, Vassallo M, Cordeschi G, Andrea SD, Necozione S, et al. (2016) Semen leukocytes and oxidative-dependent DNA damage of spermatozoa in male partners of subfertile couples with no symptoms of genital tract infection. Andrology 4(5): 808-815.

13. Majzoub A, Esteves SC, Gosálvez J, Agarwal A (2016) Specialized sperm function tests in varicocele and the future of andrology laboratory. Asian J Androl 18(2): 205-212.

14. Aitken RJ, Bronson R, Smith TB, Iuliis GN (2013) The source and significance of DNA damage in human spermatozoa; a commentary on diagnostic strategies and straw man fallacies. Mol Hum Reprod 19(8): 475-485.

15. Muratori M, Tamburrino L, Marchiani S, Cambi M, Olivito B, et al. (2015) Investigation on the origin of sperm DNA fragmentation: role of apoptosis, immaturity and oxidative stress. Mol Med 21: 109-122.

16. Hamada A, Esteves SC, Agarwal A (2013) Insight into oxidative stress in varicocele-associated male infertility: part 2. Nat Rev Urol 10(1): 26-37.

17. Majzoub A, Agarwal A (2018) Systemic review of antioxidant types and doses in male infertility: Benefits on semen parameters, advanced sperm function, assisted reproduction and live birth rate. Arab J Urol 16(1): 113-124.

18. vRoque M, Esteves C (2018) Effect of varicocele repair on DNA fragmentation: a review. Int Urol Nephrol 50(4): 583-603.
19. Parmegiani L, Cognigni G, Filicori M (2014) Sperm selection: effect on sperm DNA quality. Adv Exp Med Biol 791: 151-172.

20. Mehta A, Bolyakov A, Schlegel PN, Paduch DA (2015) Higher pregnancy rates using testicular sperm in men with severe oligospermia. Fertil Steril 104(6): 1382-1387.

21. Osman A, Alsomait H, Seshadri S, Toukhy TE, Khalaf Y (2015) The effect of DNA fragmentation on live birth rate after IVF or ICSI: A systematic review and meta-analysis. Reprod Biomed Online 30(2): 120-127.

22. Cho C, Agarwal A (2017) Role of sperm DNA fragmentation in male factor infertility: a systematic review. Arab J Urol 16(1): 21-34.

23. Milanizadeh S, Zuwarali K, Aliaghaei A, Bigdeli M (2018) Therapeutic potential of pretreatment with allograft sertoli cells transplantation in brain ischemia by improving oxidative defences. J Mol Neurosci 64(4): 533-542.

24. Bosco L, Notari T, Ruvolo G, Roccheri MC, Martino C, et al. (2018) Sperm DNA fragmentation: an early and reliable marker of air pollution. Environ Toxicol Pharmacol 58: 243-249.

25. Abhyankar N, Kathrins M, Niederberger C (2016) Use of testicular versus ejaculated sperm for intracytoplasmic sperm injection among men with cryptozoospermia: a meta-analysis. Fertil Steril 105(6): 1469-1475.

26. Arafa M, AlMalki A, AlBadr M, Burjaq H, Majzoub A, et al. (2018) ICSI outcome in patients with high DNA fragmentation: Testicular versus ejaculated spermatozoa. Andrologia 50(1): 1-6.

27. Herrero M, Lusignan M, Son W, Sabbah M, Buckett W, et al. (2019) ICSI outcomes using testicular spermatozoa in non-azoospermic couples with recurrent ICSI failure and no previous live births. Andrology 7(3): 281-287.

28. Esteves S, Martin FS, Martin PS, Schneider D, Gosalvez J (2015) Comparison of reproductive outcome in oligozoospermic men with high sperm DNA fragmentation undergoing intracytoplasmic sperm injection with ejaculated and testicular sperm. Fertil Steril 104(6): 1398-1405.

29. Zhang J, Xue H, Qiu F, Zhong J, Su J (2019) Testicular spermatozoon is superior to ejaculated spermatozoon for intracytoplasmic sperm injection to achieve pregnancy in infertile males with high sperm DNA damage. Andrologia 51(2): e13175.

30. Esteves S, Roque M, Garrido N (2018) Use of testicular sperm for intracyto plasmic sperm injection in men with high sperm DNA fragmentation: a SWOT analysis. Asian J Androl 20(1): 1-8.

31. Turek PJ (2017) Finding the fit: sperm DNA integrity testing for male infertility. Trans Androl Urol 6(Suppl 4): 379-80. 